Published in final edited form as:

Schneck, E., \& Scoppola, E. (2018). Combining Scattering and Simulation Techniques for the Study of Biomolecular Soft Interfaces. Current Opinion in Colloid \& Interface Science, 37, 88-100.

doi:10.1016/j.cocis.2018.06.008.

\title{
Combining Scattering and Simulation Techniques for the Study of Biomolecular Soft Interfaces
}

\author{
Emanuel Schneck, Ernesto Scoppola
}
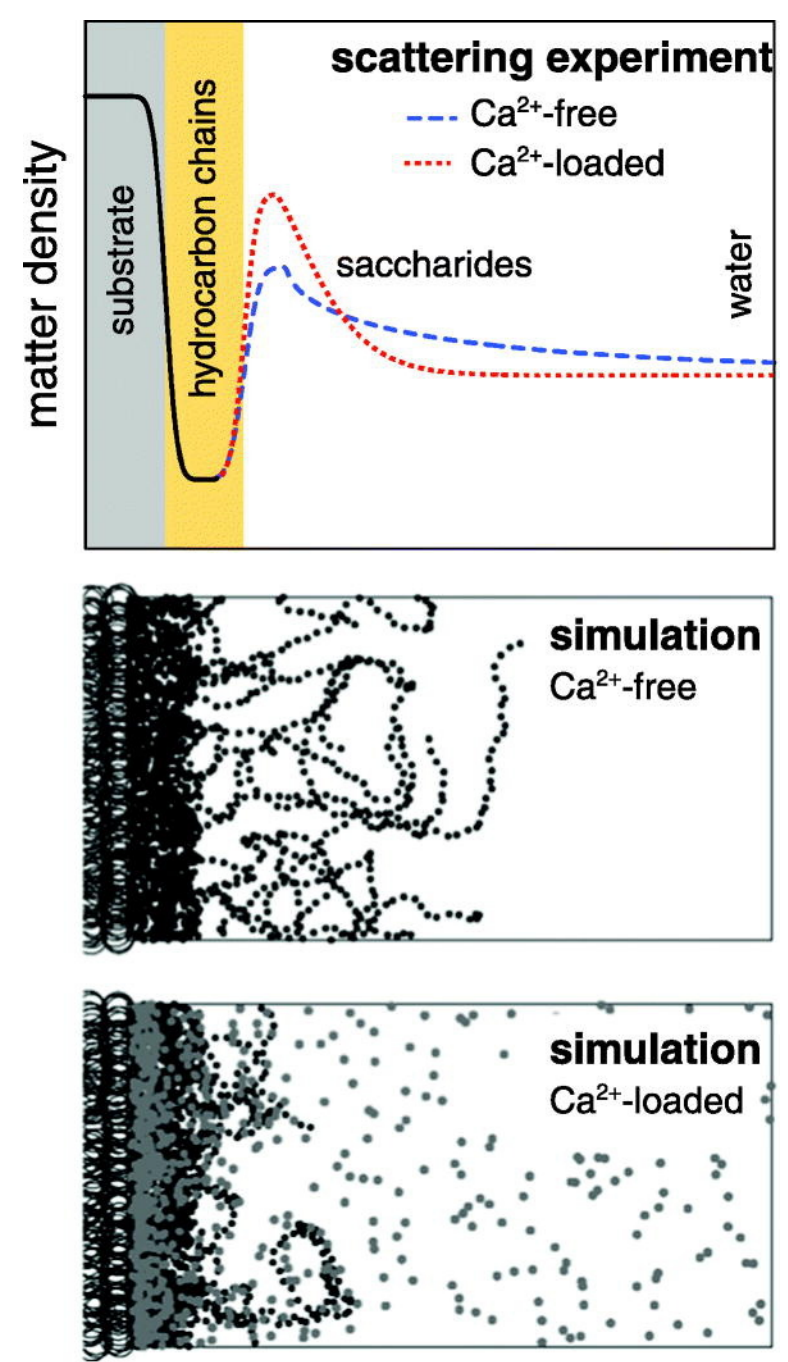

distance from interface

\author{
We present a review of the combined \\ use of computer simulations and \\ scattering techniques with x-rays and \\ neutrons for the investigation of soft \\ interfaces, whose importance in the \\ fields of biology as well as wet- and \\ biotechnology is nowadays well \\ recognized. Various scattering and \\ simulation methods are introduced and \\ recent examples of their combined or \\ synergistic use are presented. To this \\ end, the added insights generated \\ through the combination are \\ highlighted.
}




\title{
Combining scattering and computer simulation for the study of biomolecular soft interfaces
}

\author{
Ernesto Scoppola, Emanuel Schneck* \\ Biomaterials Department, Max Planck Institute of Colloids and Interfaces, \\ Am Mühlenberg 1, 14476 Potsdam (Germany)
}

\begin{abstract}
We present a review of the combined use of computer simulations and scattering techniques with x-rays and neutrons for the investigation of soft interfaces, whose importance in the fields of biology as well as wet- and biotechnology is nowadays well recognized. Various scattering and simulation methods are introduced and recent examples of their combined or synergistic use are presented. To this end, the added insights generated through the combination are highlighted.

Keywords: lipid membranes, polymer brushes, $\mathrm{x}$-ray and neutron

scattering, coarse-grained simulations, molecular dynamics

simulations
\end{abstract}

\section{Introduction}

The past decades have witnessed substantial progress in the methodologies of both computer simulations and scattering techniques with $\mathrm{x}$-rays and neutrons. Scattering experiments and computer simulations are complementary; they can both access the length scales of atoms, molecules, and supra-molecular systems, suggesting that they "naturally belong together". In fact, molecular simulations since their emergence have been bench-marked with structural data from scattering experiments [1, 2]. Bio- and soft-matter systems in particular lend them-

\footnotetext{
*Corresponding author

Email address: schneck@mpikg.mpg.de (Emanuel Schneck )
} 
selves towards combined scattering/simulation approaches, because equilibrium structures on the nanometer scale are typically formed on computationallyaccessible time scales. For bulk soft-matter systems, combining scattering and simulations has a long tradition [3, 4, 5, 6, 7, 8, 9, 10, 11. When it comes to planar interfaces, even more detailed insight can be obtained because structural information in in-plane and out-of-plane directions can be disentangled (see Fig. 1). To this end, scattering and simulations were combined, for instance, to investigate the configuration of water near hydrophilic [12] and hydrophobic [13] solid surfaces.

For biomolecular soft interfaces the potential of combined scattering/simulation approaches has been exploited only more recently. Soft interfaces in the form of interfacial molecular layers are a major component of all biological matter and also centrally involved in numerous wet- and biotechnological applications. For example, the architecture of functional biomolecular assemblies like biomembranes, the performance of bio-compatible functionalization, or the performance of lubricants depend on the way molecules and ions self-organize at the interface between two extended media. Processes of interest often involve the spatial organization and dynamics of interfacial molecules, interfacial adsorption of ions or molecules, and the response of molecular conformations to external stimuli, among many others.

In the following, we discuss combinations of scattering experiments and computer simulations for the study of biomolecular interfaces. In the first section we review methodology, state-of-art, and fields of application of scattering and simulation techniques on their own. Then we review the more-or-less recent developments in the combination of the two. Examples include studies involving soft interfaces of biological relevance but also related studies from other fields of soft matter interface science. The largest part is concerned with membranes and simplified mimics thereof, because they are the most important and most intensely studied biological interfaces. Finally we give an outlook to promising future directions exploiting the complementarity of scattering and simulation techniques. 


\section{Methodological background}

\subsection{X-ray and neutron scattering from soft and biomolecular interfaces}

X-ray and neutron scattering probe soft interfaces with the sub-nanometer spatial resolution required to determine molecular-level details. These scattering techniques can be applied in a wide pressure and temperature range and provide sample-averaged structural information. While x-ray scattering typically offers higher spatial resolution, neutron scattering, apart from being truly non-destructive, has the unique advantage of contrast variation: chemical components of interest can be highlighted by isotopic substitution (e.g., by replacing hydrogen with deuterium) without changing their chemistry. Small-angle x-ray and neutron scattering as well as wide-angle x-ray scattering, SAXS, SANS, and WAXS, respectively, have been used to investigate randomly-oriented interfaces in bulk oil/water emulsions or in dispersions of multilamellar vesicles [4, 5, 6, 8, 9, 11]. Such measurements yield insights into short-range molecular ordering, the size distribution and shape of colloidal objects, and under certain conditions also into the matter distributions across their interfaces. However, scattering techniques for the study of interfaces unfold their full potential only when samples possess planar geometry, allowing the specific probing of structures perpendicular and parallel to the interface (see Fig. 1). While grazing-incidence small-angle scattering with x-rays (GISAXS) or neutrons (GISANS), and as well as grazing-incidence x-ray diffraction (GIXD) yield information on the in-plane structure of an interface (Fig. 1B), specular x-ray reflectometry (XRR) and neutron reflectometry (NR) reveal matter density profiles perpendicular to the interface in terms of interfacial scattering length density (SLD) profiles $\rho(z)$ (Fig. 1A left) [14, 15]. On the other hand, off-specular scattering with x-rays and neutrons, termed OSXS and OSNS in the following, are means to characterize the interface topography $(h(x)$, see Fig. $1 \mathrm{C}$ top) in terms of height-height correlation functions $(C(r)$, see Fig. 1C bottom) [14, 15]. $\mathrm{X}$-ray fluorescence techniques are suited to determine element-specific density profiles $c(z)$ across an interface at sub-nm resolution [16] (Fig. 1A, right). Fi- 
nally, inelastic neutron scattering provides insights into the dynamics of soft interfaces [17, 18]. In the following paragraphs we discuss a number of experimental studies which illustrate the performance of the scattering methods introduced above for the characterization of soft and biomolecular interfaces.

\subsubsection{Out-of-plane structures}

Matter distributions. Surfaces displaying grafted polymers in good solvents are prototypical examples of soft interfaces. NR was used to characterize the conformation of polymer brushes and thereby, for the first time, allowed confronting the predictions of existing brush theories with reality [19, 20, 21, 22, 23, In the biological domain, SAXS, SANS, XRR and NR have been intensively used to characterize models of biomembranes and cell surfaces. Impressive structural detail has been achieved for lipid bilayers [24, 25]. In fact, the structural parameters deduced in these experimental studies are commonly used to benchmark lipid force fields in computer simulations [26]. XRR and NR revealed also other aspects of lipid membranes, such as the architecture of floating lipid bilayers [27, the configuration of membrane-associated proteins [28, 29, 30, 31, and the conformation of lipopolysaccharides in models of bacterial outer surfaces [32, 33.

Charges, ions, and element distributions. X-ray fluorescence in a total reflection configuration (TRXF) was used to determine excesses of counter- and co-ions at a charged surfactant monolayer at an air water interface, where the monolayer charge was quantified as a function of the lateral compression [34. This strategy was later extended to bare air/water interfaces, in order to determine the specific preferential depletion and accumulation of various ion types [35], and to charged lipopolysaccharide monolayers mimicking bacterial surfaces [36]. More recently, x-ray fluorescence in a standing-wave configuration (SWXF [37]) was optimized for lighter chemical elements in order to localize the biologically relevant chemical elements $\mathrm{S}$ and $\mathrm{P}$ in biomolecular layers with atom-scale resolution [38]. 


\subsubsection{In-plane structures}

GIXD was used to comprehensively characterize the in-plane structural ordering of lipid and surfactant monolayers at air/water interfaces [39, 40] on the $\AA$ scale. The obtained information includes the hydrocarbon chain $2 \mathrm{D}$ crystalline unit cell dimensions ( $a$, and $b$, see Fig. 1B), the chain tilt angle $(\theta$, see Fig. 1B) with respect to the surface normal $(z)$ and its direction, as well as the thickness of the crystalline layer. More recently, GIXD enabled the complete reconstruction of the complex unit cell of glycolipid monolayers exhibiting chain and headgroup crystalline ordering [41. On a slightly larger in-plane length scale of several nanometers, the same technique revealed the lateral organization of proteins attached to a lipid monolayer with lipid anchors [42] and the lateral superstructure of lipopolymer monolayers [43].

\subsubsection{Interface topography}

OSXS was used to characterize the time-averaged interfacial topography (Fig. 1C) associated with the thermal fluctuations of lipid bilayers floating above planar solid supports [27. This allowed reconstructing thermodynamic and mechanical bilayer properties like tension, bending elasticity, and interaction modulus with the supporting surface. Based on the same principles, analysis of the OSXS or OSNS intensities from solid-supported oriented membrane multilayers provided comprehensive insights into membrane fluctuation self- and cross-correlation functions and the underlying mechanical properties [44, 45].

\subsubsection{Interface dynamics}

The undulatory excitations of stacked lipid bilayers were investigated by inelastic neutron scattering [46] in a neutron spin echo (NSE) configuration, which allows probing the small energy changes associated with the comparatively slow membrane undulations [18]. The dynamics in membranes on smaller length and time scales, including headgroup and tail mobility [47] and correlated lipid dynamics [48, has been investigated by the time-of-flight (TOF) and backscattering methods, which are sensitive to the larger energy transfers associated 



Figure 1: Structural information accessible by x-ray and neutron scattering from an interface. (A) Out-of-plane matter distributions in terms of the interfacial SLD profile $\rho(z)$ (left) and the individual distributions $c(z)$ of chemical elements (right). (B) In-plane structures in terms of molecular 2D-periodic arrangements characterized by the unit cell dimensions $a$ and $b$ and the molecular tilt angle $\theta$. (C) Interface topography in terms of local height variations $h(x)$ and their in-plane correlation $C(r)$.

with faster processes [17, 18].

\subsection{Computer simulations of soft and biomolecular interfaces}

Computer simulations have proven to be powerful tools for the study of soft interfaces on various length and time scales. Large-scale aspects like surface shapes on the micrometer scale have traditionally been described with continuum-theoretical simulations (see Fig. 2A) in terms of interfacial tensions, bending rigidities, and interaction potentials [49, 50, 51]. A considerable level of molecular detail is represented in coarse-grained (CG) simulations (see Fig. 2B) in which molecular moieties interact via effective pair potentials. In CG simulations the solvents can either be implicit [52, 53, or represented by groups of solvent molecules [54]. Length scales of up to hundred nanometers are accessible at time scales of up to milliseconds. Higher spatial resolution is accessible with atomistic molecular dynamics (MD) simulations (see Fig. 2C), which capture atom-level chemical details including all solvent molecules. However, they are computationally more demanding and therefore today usually limited to length scales of up to several tens of nanometers and time scales of up to several 
microseconds. In the following paragraphs we discuss a number of simulation studies which illustrate the state-of-art of the simulation techniques introduced above for the investigation of soft and biomolecular interfaces. We leave abinitio simulations [55] aside, because their combination with x-ray or neutron scattering from soft matter and interfaces is not very common.

Continuum-theoretical simulations. Continuum-theoretical simulations based on finite volume methods have been employed to describe droplet coalescence in water-in-oil emulsions, depending on various parameters including the interfacial tension [51]. With the help of Monte-Carlo (MC) simulations, Hu et al. investigated the morphologies of vesicles comprising multiple membrane domains [50], see Fig. 2A (top). The vesicle surfaces were discretized and bending and interaction energies were associated to each configuration. In another study, the dynamic topography of lipid bilayers thermally fluctuating near a planar surface and displaying specific adhesion molecules was characterized in MC simulations of discretized yet interconnected membrane segments [49].

Coarse-grained (CG) simulations. CG simulations of surface-grafted polymer brushes [56, 57, 58, 59] were used to test the predictions of analytical brush theories such as the Alexander-de-Gennes (AdG) and self-consistent field (SCF) models. The simulation studies used implicit or explicit solvents and various bead-spring models of the polymer chains, differing in the treatment of nonbonded interactions. For suitable parameters the resulting brush profiles exhibited satisfactory agreement with those obtained in NR experiments [19, 20, 21, 22. CG simulations have also contributed to our knowledge of the behavior of biological interfaces. By using CG representations of lipids [54 (see Fig. 2B), various aspects of biological membranes have been investigated, ranging from the distribution of lipid species in multi-component bilayers [60] to the estimation of membrane bending rigidities [61] and the budding and fusion of lipid vesicles [62]. The effect of divalent cations on the interaction of antimicrobial peptides with Gram-negative bacteria was studied with the help of MC simulations based on a $\mathrm{CG}$ representation of the bacterial surface featuring negatively 

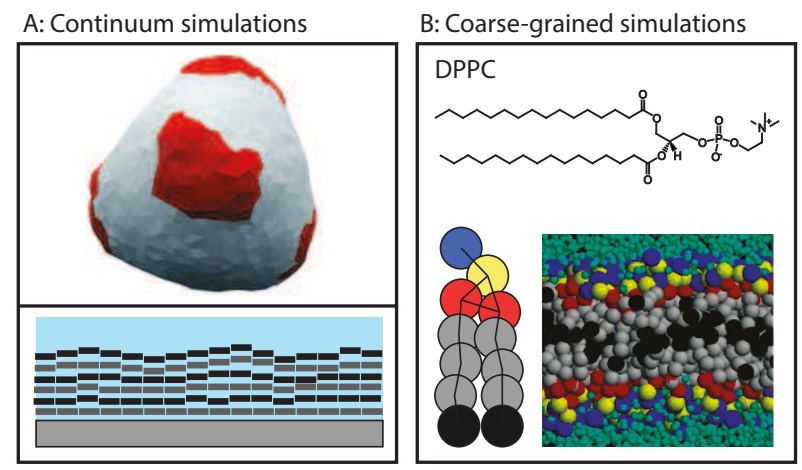

C: Atomistic simulations

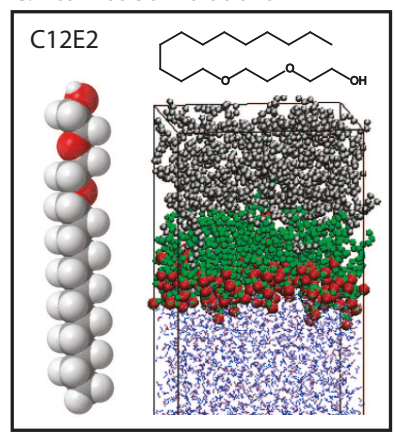

Figure 2: Schematic illustration of various types of computer simulations. (A) Continuumtheoretical simulations based on discretized interface or layer elements. Top: lipid vesicle. From Hu et al. [50. Bottom: a stack of interacting molecular layers. (B) Coarse-grained simulations in which chemical moieties are represented as particles interacting via effective potentials. From Marrink et al. [54. (C) Atomistic simulations featuring atom-scale chemical detail. From Chanda et al. 63.

charged oligo- and polysaccharides [52].

Atomistic simulations. Atomistic MD simulations were used to investigate the configuration of surfactants at air/water and oil/water interfaces [63, see Fig. 2C. This technique also provided insight into ion-specificity in the interaction of ions with the hydrophobic air/water interface, since, in contrast to CG simulations, atomistic simulations capture the details of the hydration shell of each ion type [64]. Over the last decade, atomistic MD simulations have been extensively employed for the study of lipid membranes and are now able to reproduce, for instance, the structure of both fluid and gel phase lipid bilayers [26], the behavior of lipid mixtures [60], and the difference in the interaction between phospholipid glycolipid membranes [65]. Recent studies also addressed the configuration of membrane proteins and their interaction with multi-component membranes [60] and the adsorption of peptides onto membranes [66]. 


\section{Combination of scattering and simulation methods}

Motivations to combine scattering, like all experimental techniques [67, with computer simulations can be diverse. At first, experimental results can be used to verify simulations or to adjust simulation parameters like inter-particle potentials or conformational degrees of freedom [68]. Once the simulations reproduce a sufficient number of experimental observables, like the average area per molecule or the layer thickness, they will likely also correctly capture aspects which are not experimentally accessible. Second, experiments can provide initial configurations for simulations, which would not have been obtained by the simulations alone, due to the slow timescales of the emergence of these configurations/structures, i.e., due to high free-energy barriers between various (meta-)stable configurations. Finally, simulations can be used to exclude, on physical grounds, certain lines of interpretation of ambiguous experimental data.

A straight-forward approach to combine scattering and simulation data is to compute the time-averaged scattering intensity $S(q)$ corresponding to the content of the simulation box, based on pre-defined scattering form factors of the individual particles. This strategy was used, for example, to test the quality of various water models for atomistic MD simulations [69. Good qualitative agreement with SAXS/WAXS from bulk water was found for all water models investigated, and it was concluded that the scattering features at low $q$ are mainly correlated to the spatial density correlation of the water model. In a recent review on combined SAXS/MD studies of biomolecular assemblies [70] it was concluded that MD simulations are suited to extract the maximum information content from high-resolution SAXS data. The highest level of synergy between scattering and simulations is today probably reached with the help of so-called empirical potential structural refinement (EPSR) [71]. In this method, $S(q)$ as computed from the simulations is compared to the experimental data and refined via addition of empirical inter-particle potentials until optimal agreement is achieved. EPSR has been successfully applied to determine structural 
features of liquids [71] and amorphous materials [72].

\subsection{Soft interfaces}

Scattering and simulations have been combined for the study of soft interfaces of both biological and technological relevance. Before we turn to the biomolecular interfaces, we give a brief overview of important work on nonbiological systems, to which the same methodological concepts apply.

Fukuto et al. investigated the interface between water and alkane oil, two immiscible liquids, by combining XRR and atomistic MD simulations [73. Traditionally, the laterally averaged electron density profile has been considered to vary monotonically between those of the two bulk media over a width $\sigma_{C W}$ solely dictated by the thermal capillary waves $(\mathrm{CW})$. However, previous XRR measurements suggested the existence of a depletion layer of highly-debated origin. In the study by Fukuto et al., XRR measurements yielded the exact interfacial roughness, while complementary MD simulations revealed an alignment of alkane molecules at the interface and, in turn, an enrichment of methyl groups with low electron density. The corresponding equivalent depletion layer was found to be at the order of $0.5 \AA$. With this notion, CW model and XRR results were finally reconciled.

Ferru et al. used a combination of SAXS/WAXS and MD simulations to investigate the molecular and supra-molecular ordering of extractant molecules in the organic phase of a liquid/liquid extraction system [11]. For one extractant type, the $S(q)$ predicted by the MD simulations was in good agreement with the experimental SAXS data and allowed interpreting them in an unambiguous manner. For another type, comparison of SAXS with the simulated $S(q)$ allowed attributing a distinct intensity maximum to the correlation distance between alcohol $\mathrm{OH}$ groups.

In a study by Schöttl et al., SAXS and atomistic MD were combined to investigate octanol/ethanol/water ternary solutions [74. $S(q)$ from experiment and simulation exhibited excellent agreement including a pronounced shift of an intermolecular correlation peak upon increasing the water content. The simula- 
tions revealed the sub-structure of octanol-rich domains, in which ethanol was found to be enriched at the surface.

Hiotelis et al. combined NR at the solid/liquid interface with bond-fluctuation coarse-grained Monte-Carlo (BFMC) simulations to study surface-end-grafted polymer brushes formed by centrally adsorbed star polymers [23], see Fig. 3A. Experiments and simulations yielded consistent polymer volume fraction profiles, which are shown in Fig. 3B and 3C, respectively. With that, the experiments served for the validation of the BFMC simulation technique, which in turn, provided qualitative insight into the adsorption kinetics. The presence of multiple arms in each star-shaped macromolecule resulted in a significant decrease of the surface binding and an increase of the mean distance ( $S$, see Fig. 3A) between anchoring points due to intermolecular repulsive interactions. A review article about the simulation-assisted interpretation of experimental data on polymer brushes has been provided by Binder and Milchev [75.

In two independent experimental and simulation-based studies, Padmanabhan et al. [35] and Horinek et al. [64], respectively, investigated the distributions of ions at air/water interfaces. The experimental study employed XRR and TRXF to quantify the interfacial depletion or accumulation of various ion species, while the simulation study employed atomistic MD. Both approaches consistently found that iodine exhibits preferential interactions with the interface, and that lithium exhibits anomalous behavior. Although the two studies were independent, together they clearly demonstrate the complementarity of scattering and simulation techniques and the great potential of their combined use when it comes to ion-specific effects.

\subsection{Biomolecular interfaces}

As pointed out in the Introduction, the by far largest body of work on biomolecular interfaces has been concerned with lipid layers and biological membranes. Peters et al. combined GIXD experiments with atomistic MD simulations to determine the structural ordering of dipalmitoylglycerol monolayers at air/water interfaces depending on the area per molecule $A_{m}$ [76]. Experiments 

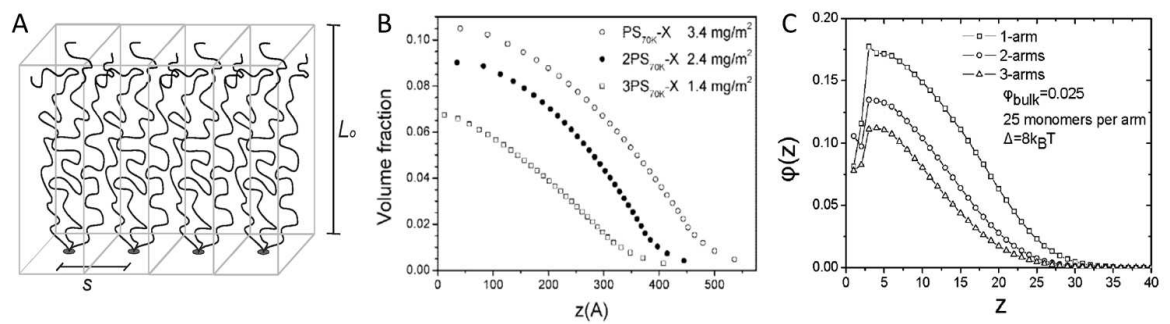

Figure 3: From a combined NR and CGMC study on surface-grafted polymer brushes: (A) Schematic illustration of centrally surface-adsorbed star polymers with average distance $S$ forming a brush with extension $L_{0}$. (B) and (C) Polymer volume fraction profiles obtained in experiments and simulations, respectively. From Hiotelis et al. 23.

and simulations consistently revealed two phase transitions at $A_{m}=38.3 \AA^{2}$ and $A_{m}=39.8 \AA^{2}$, respectively, see Fig. $4 \mathrm{~A}$. The intensity profiles of various diffraction peaks in experiments (Fig. 4B) and simulations (Fig. 4C) were found to be in good agreement and demonstrated that the transition at $A_{m}=39.8 \AA^{2}$ is due a change in the alkyl chain tilt angle (see $\theta$ in Fig. 1B). Simulation analysis further revealed that the transition $A_{m}=38.3 \AA^{2}$ involves a reorientation of the headgroups. The same GIXD/MD combined approach would be desirable also to elucidate more complex monolayer structures like the subgel structures of glycolipids reported by Stefaniu et al [41. However, since crystal formation may be too slow to be observed on the time scales covered by MD simulations today, pre-arranged initial configurations based on the GIXD data may be required (see further below).

By using XRR on solid-supported phospholipid multi-bilayers, Salditt et al. determined the electron density profile across the bilayers [77 and found good agreement with the corresponding profiles obtained in MD simulations. Later on, neutron diffraction and MD simulations were combined to scrutinize the orientation of cholesterol molecules in lipid bilayers depending on the fraction of lipids with saturated (DMPC), unsaturated (POPC), and poly-unsaturated (PUFA) fatty acids [78]. Cholesterol was found lying parallel in the center of 

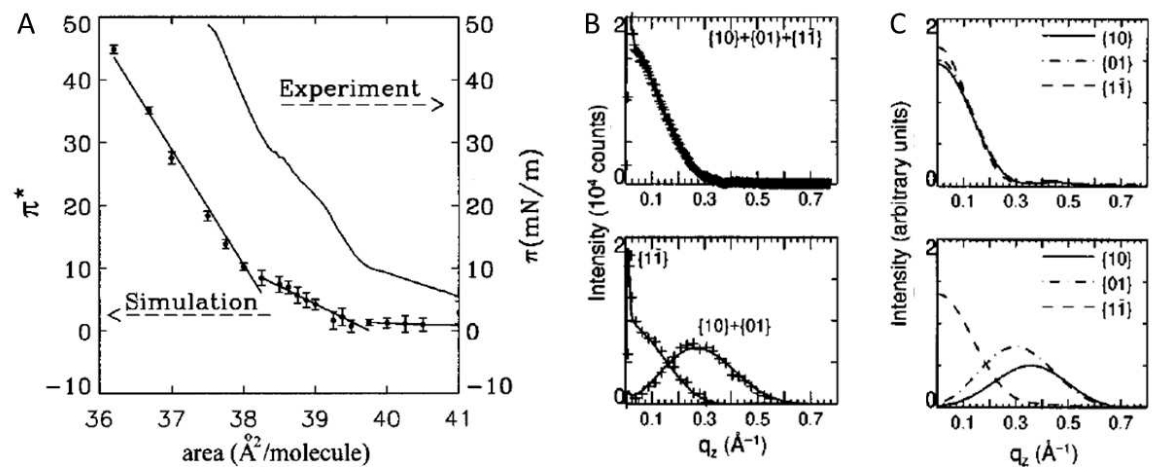

Figure 4: From a combined GIXD and MD study on dipalmitoylglycerol monolayers at air/water interfaces: (A) Pressure-area isotherms as obtained in the simulations (left ordinate) and as obtained in the experiment (right ordinate). (B) and (C) Diffraction intensities from experiments and simulations, respectively, at $A_{m}=39.6 \AA^{2}$ (top) and $A_{m}=40.5 \AA^{2}$ (bottom). From Peters et al. 76].

a PUFA bilayer at low POPC concentrations. Above a critical POPC concentration it reorients to an upright configuration. This critical concentration is lower when POPC is replaced with DMPC. The simulations revealed (i) the formation of DMPC-rich domains where cholesterol is located preferentially in its upright orientation and (ii) domains depleted of DMPC where cholesterol is found mostly in the bilayer center. More recently, Jämbeck et al. developed an all-atom MD force field for saturated phospholipids in both fluid and gel membranes [26]. The performance of the force field in reproducing x-ray scattering form factors $|F(q)|$ (in analogy to $S(q)$ introduced further above) was an important validation criterion. A combination of atomistic MD simulations and neutron diffraction was later exploited by Kanduc et al. to investigate hydration forces between phospholipid and glycolipid membranes [65]. The simulations allowed precisely determining the partial molecular volume of water which, in turn, can be used to reinterpret the strength of the equivalent pressure for a given water chemical potential in the scattering experiments.

By combining CGMC simulations with a reflectometry-equivalent x-ray scattering technique (termed GIXOS), Oliveira et al. studied lipopolysaccharide 

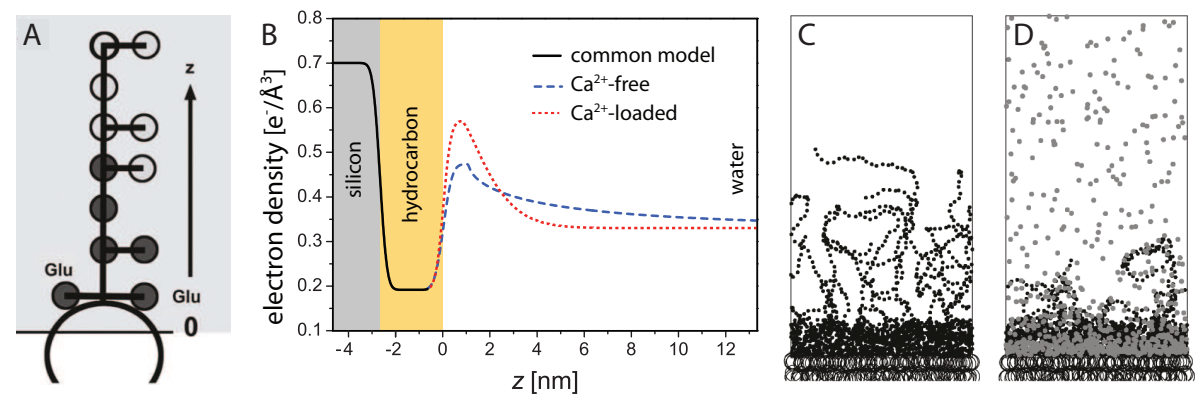

Figure 5: From combined XRR and CGMC simulation studies on planar lipopolysaccharide (LPS) monolayers as mimics of bacterial outer surfaces: (A) Schematic illustration of the computer model of the LPS molecules. O-side chains (see main text) are not shown. (B) Electron density profile (proportional to the SLD profile discussed above and schematically illustrated in Fig. 1A left) of a solid-supported LPS monolayer bearing negatively charged Oside chains in the absence and presence of calcium ions. (C) and (D) Simulation snapshots in the absence and presence, respectively, of calcium ions. From Oliveira et al. 79] and Schneck et al. [33].

(LPS) monolayers at air/water interfaces as mimics of the outer surface of Gramnegative bacterial membranes in the absence and presence of divalent cations and antimicrobial peptides [79, 40. In the simulation model (Fig. 5A), saccharides and peptide amino acids were represented as electrically neutral or charged spheres connected via stretchable bonds, water was implicit, and ions were represented by small, charged spheres. Both experiments and simulations revealed that divalent cations induce a compaction of the LPS headgroups and render the monolayer impermeable to the peptides. XRR and CGMC simulations also consistently showed that the conformation of more complex LPS molecules featuring long, negatively charged polysaccharides (termed O-side chains), is even more sensitive to the presence of divalent cations [33], see Fig. 5 B-D. The same type of simulations later served to interpret ion-specific TRXF data evidencing the near-complete displacement of monovalent cations by divalent cations from the negatively charged LPS core saccharides [36].

Synergistic use of SAXS/SANS and implicit-solvent CG simulations was made by Midtgaard et al. in order to investigate the self assembly and struc- 
ture of lipid/peptide nanodiscs in aqueous dispersion [53]. The structural model for the analysis of the scattering data was refined based on the information obtained in the simulations, which predicted polydispersity in the disc size and the presence of peptide trimers, consistently with the experimental data. In a study by Constantin et al., it was a continuum model of solid-supported lipid multi-bilayers (see schematic illustration in Fig. $2 \mathrm{~A}$ bottom) that helped the authors interpreting their XRR experimental data [80]. Focus of their study were the correlated thermal fluctuations in lipid membrane stacks. Taking an inverse strategy, Coppock et al. used x-ray scattering data as basis for the initial configuration of ordered lipids in atomistic MD simulations of gel-phase membranes 81 .

Klauda et al. proposed the analysis of bilayer x-ray diffraction data with the help of atomistic MD simulations [82]. By systematically varying in the simulations the area per lipid molecule $A_{m}$, which is the observable most sensitive to the force field, a spectrum of physically meaningful and self-consistent bilayer structures can be generated. The resulting electron density profiles are shown in Fig. 6A. The associated form factor $|F(q)|$ can then be computed (see Fig. 6B) and compared to the experimental data (Fig. 6C). The bilayer structure corresponding to the best-matching $A_{m}$ can be considered a high-quality representation of the experimental system, because the simulation model imposes meaningful physical constraints. With a similar motivation, Fogarty et al. later on developed an atomic density profile (ADP) model for the analysis of scattering data from lipid bilayers [83, along the line introduced earlier for the distributions of multiatomic molecule fragments by Wiener and White [24]. In the model by Fogarty et al., which is readily adaptable also to other soft or biological interfaces, the ADPs are subject to physical constraints like bond lengths, the volumes of molecular moieties, and others, some of which are imposed by penalty terms. A review article on the co-refinement of phospholipid membrane structures based on simulations and experimental data was recently published by Ollila and Pabst $[84$. 

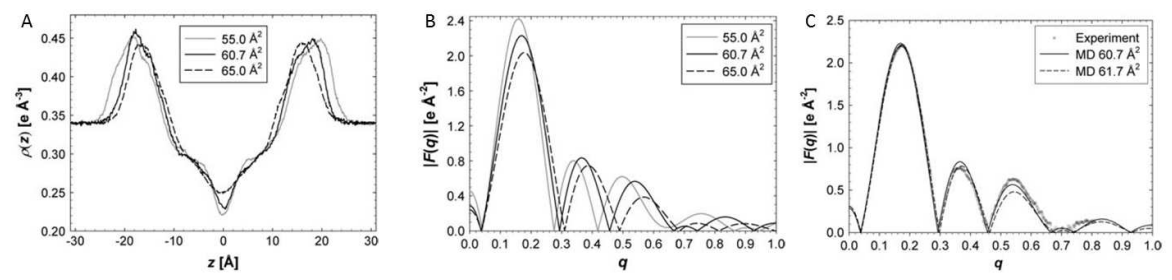

Figure 6: From a combined x-ray diffraction and MD simulation study on lipid bilayers: (A) Electron density profiles along the bilayer normal $(z)$ obtained in the simulations for $A_{m}=55 \AA^{2}, A_{m}=60.7 \AA^{2}$ and $A_{m}=65 \AA^{2}$. (B) Corresponding form factors $|F(q)|$. (C) Comparison of the experimental and simulated form factors at $A_{m} \approx 61 \AA^{2}$. From Klauda et al. 82 .

Aspects of membrane dynamics were investigated by Hub et al. [85. They combined MD simulations and scattering methods including inelastic neutron scattering to characterize the dynamics of lipids in bilayers as contained in the so-called intermediate scattering function $S(q, \omega)$. Satisfactory agreement between simulations and experiments was found on the larger length scales (lower $q$ ), while deviations at shorter length scales (higher $q$ ) were attributed to the united-atom representation of $\mathrm{CH}_{2}$ and $\mathrm{CH}_{3}$ groups in the simulation force field used. Nickels et al. combined SANS, inelastic neutron scattering, and atomistic MD simulations to characterize nanodomains in unilamellar vesicles, whose lipid composition mimics that of mammalian plasma membranes [86]. SANS with contrast matching and the simulations consistently showed that the domains are in-register across the bilayer leaflets. Inelastic neutron scattering further revealed that the bending modulus of the nanoscopic domains differs from that of the surrounding continuous phase. Interestingly, the motion of the nanodomains is enhanced due to a flexible region between the two phases, as consistently found in experiments and simulations.

A comparatively complex biology-related system was studied by Shenoy et al. [28, 29], who investigated the configuration of a protein (PTEN phosphatase) associated with a negatively-charged lipid bilayer, see Fig. 7A. NR was used to obtain information about the protein density on the bilayer surface as well as the 

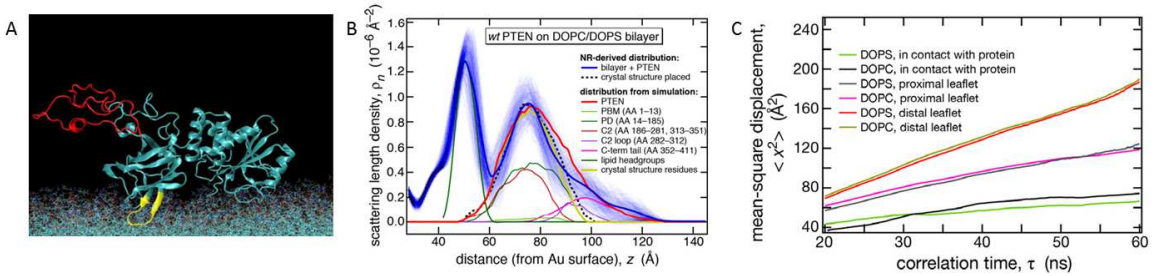

Figure 7: From a combined NR and MD simulation study on membrane-associated proteins (PTEN phosphatase): (A) Simulation snapshot the protein configuration on the membrane. (B) Comparison between experimental SLD profile (blue line surrounded by confidence interval) and the contribution of various chemical components as obtained in the simulations. (C) Mean-square displacement of various lipid subgroups as obtained in the simulations. From Shenoy et al. 29].

neutron SLD profile across the interface (see Fig. 7B). MD simulations, on the other hand, served to interpret the SLD profile in terms of the distributions and contributions of various protein subunits and membrane components. Moreover, the simulations provided insights into the dynamics of the protein and of various lipid subgroups (see Fig. 7C). More recently, Tietjen et al. interpreted XRR experiments on membrane-bound proteins (Tim: T cell immunoglobulin mucindomain) by using a-priori protein structural knowledge from crystallography and/or NMR [30, 31]. In the course of the data analysis, the protein structure and conformation with respect to the membrane was refined with the help of MD simulations. Indeed, the refined structure yielded significantly improved fits to the XRR data. Moreover it provided a detailed model of membrane-bound Tim, comprising two distinct states with different insertion depths and positions of a calcium ion pocket.

\section{Concluding Remarks}

Concerning work on soft and biomolecular interfaces, scattering and simulation methods have so far been combined mostly on a comparative level, revealing consistencies and/or deviations between experiments and simulations. Fewer studies have made more synergistic use of the combination, by either feed- 
ing information from scattering experiments into simulations or by interpreting scattering data with the help of computer models. Scattering and simulation methods become truly complementary when the phenomena under investigation involve molecular exchange between interfaces and bulk media. Namely, the simulations alone are essentially unable to handle this manifold-Grand-Canonical problem to work out the molecular composition at an interface. But the latter can be solved in a straight-forward manner with scattering experiments like NR with contrast variation. With the correct interfacial molecular stoichiometry at hand, the simulations can then provide structural and mechanistic details with a resolution far beyond that of the experiments.

Another very promising direction for future studies is the "EPSR-like" modeling of scattering signals from soft or biomolecular interfaces based on adjustable bonded and non-bonded interactions in CG or atomistic simulations. Such approaches will likely find broader use with the advent of ever more powerful computing infrastructure available to the scientific community.

One of the authors of this review article was once advised by a scientific mentor: "Don't fit structure. Fit physics!" (or words to that effect). In a nutshell this is what the combination of scattering and simulation methods ultimately offers.

\section{Acknowledgement}

The authors thank the Max Planck Society (MPG) for financial support. E. Schneck acknowledges support from an Emmy-Noether grant (SCHN 1396/1) of the German Research Foundation (DFG).

\section{References}

[1] F. H. Stillinger, A. Rahman, Improved simulation of liquid water by molecular dynamics, The Journal of Chemical Physics 60 (4) (1974) 1545-1557.

[2] W. Streett, D. Tildesley, Computer simulations of polyatomic molecules ii. molecular dynamics studies of diatomic liquids with atom-atom and 
quadrupole-quadrupole potentials, Proc. R. Soc. Lond. A 355 (1681) (1977) 239-266.

[3] W. Dietz, K. Heinzinger, Structure of liquid chloroform. a comparison between computer simulation and neutron scattering results, Berichte der Bunsengesellschaft für physikalische Chemie 88 (6) (1984) 543-546.

[4] A. Hasmy, M. Foret, J. Pelous, R. Jullien, Small-angle neutron-scattering investigation of short-range correlations in fractal aerogels: Simulations and experiments, Physical Review B 48 (13) (1993) 9345.

[5] R. Scherrenberg, B. Coussens, P. Van Vliet, G. Edouard, J. Brackman, E. De Brabander, K. Mortensen, The molecular characteristics of poly (propyleneimine) dendrimers as studied with small-angle neutron scattering, viscosimetry, and molecular dynamics, Macromolecules 31 (2) (1998) $456-461$.

[6] S. Rathgeber, T. Pakula, V. Urban, Structure of star-burst dendrimers: A comparison between small angle x-ray scattering and computer simulation results, The Journal of chemical physics 121 (8) (2004) 3840-3853.

[7] S. Rathgeber, T. Pakula, A. Wilk, K. Matyjaszewski, H.-i. Lee, K. L. Beers, Bottle-brush macromolecules in solution: Comparison between results obtained from scattering experiments and computer simulations, Polymer 47 (20) (2006) 7318-7327.

[8] G. N. Clark, G. L. Hura, J. Teixeira, A. K. Soper, T. Head-Gordon, Smallangle scattering and the structure of ambient liquid water, Proceedings of the National Academy of Sciences 107 (32) (2010) 14003-14007.

[9] S. Li, J. L. Bañuelos, J. Guo, L. Anovitz, G. Rother, R. W. Shaw, P. C. Hillesheim, S. Dai, G. A. Baker, P. T. Cummings, Alkyl chain length and temperature effects on structural properties of pyrrolidinium-based ionic liquids: a combined atomistic simulation and small-angle x-ray scattering study, The Journal of Physical Chemistry Letters 3 (1) (2011) 125-130. 
[10] E. Scoppola, A. Sodo, S. E. McLain, M. A. Ricci, F. Bruni, Waterpeptide site-specific interactions: A structural study on the hydration of glutathione, Biophysical journal 106 (8) (2014) 1701-1709.

[11] G. Ferru, D. Gomes Rodrigues, L. Berthon, O. Diat, P. Bauduin, P. Guilbaud, Elucidation of the structure of organic solutions in solvent extraction by combining molecular dynamics and x-ray scattering, Angewandte Chemie International Edition 53 (21) (2014) 5346-5350, (*) Combination of SAXS experiments and MD simulations reveals structural details of a liquid/liquid extraction system.

[12] M. Ricci, V. Tudisca, F. Bruni, R. Mancinelli, E. Scoppola, R. Angelini, B. Ruzicka, A. Soper, The structure of water near a charged crystalline surface, Journal of Non-Crystalline Solids 407 (2015) 418-422.

[13] M. Mezger, F. Sedlmeier, D. Horinek, H. Reichert, D. Pontoni, H. Dosch, On the origin of the hydrophobic water gap: an x-ray reflectivity and md simulation study, Journal of the American Chemical Society 132 (19) (2010) $6735-6741$.

[14] J. Als-Nielsen, D. McMorrow, Elements of modern X-ray physics, John Wiley \& Sons, 2011.

[15] J. Daillant, A. Gibaud, X-ray and Neutron Reflectivity - Principles and Applications, Berlin: Springer, 1999.

[16] E. Schneck, B. Demé, Structural characterization of soft interfaces by standing-wave fluorescence with x-rays and neutrons, Current Opinion in Colloid \& Interface Science 20 (4) (2015) 244-252.

[17] V. G. Sakai, A. Arbe, Quasielastic neutron scattering in soft matter, Current Opinion in Colloid \& Interface Science 14 (6) (2009) 381-390.

[18] I. Hoffmann, Neutrons for the study of dynamics in soft matter systems, Colloid and Polymer Science 292 (9) (2014) 2053-2069. 
[19] J. Field, C. Toprakcioglu, R. Ball, H. Stanley, L. Dai, W. Barford, J. Penfold, G. Smith, W. Hamilton, Determination of end-adsorbed polymer density profiles by neutron reflectometry, Macromolecules 25 (1) (1992) 434439.

[20] A. Karim, S. Satija, J. Douglas, J. Ankner, L. Fetters, Neutron reflectivity study of the density profile of a model end-grafted polymer brush: influence of solvent quality, Physical review letters 73 (25) (1994) 3407.

[21] D. Perahia, D. Wiesler, S. Satija, L. Fetters, S. Sinha, S. Milner, Neutron reflectivity of end-grafted polymers: Concentration and solvent quality dependence in equilibrium conditions, Physical review letters 72 (1) (1994) 100.

[22] J. Majewski, T. Kuhl, M. Gerstenberg, J. Israelachvili, G. Smith, Structure of phospholipid monolayers containing poly (ethylene glycol) lipids at the air- water interface, The Journal of Physical Chemistry B 101 (16) (1997) $3122-3129$.

[23] I. Hiotelis, A. G. Koutsioubas, N. Spiliopoulos, D. L. Anastassopoulos, A. A. Vradis, C. Toprakcioglu, A. Menelle, G. Sakellariou, N. Hadjichristidis, Neutron reflectivity and computer simulation studies of selfassembled brushes formed by centrally adsorbed star polymers, Macromolecules 41 (20) (2008) 7648-7655, (*) Combination of NR experiments and CGMC simulations provides insight into conformation and adsorption kinetics of polymer brushes.

[24] M. C. Wiener, S. H. White, Structure of a fluid dioleoylphosphatidylcholine bilayer determined by joint refinement of x-ray and neutron diffraction data. iii. complete structure, Biophysical journal 61 (2) (1992) 434-447.

[25] N. Kučerka, M.-P. Nieh, J. Katsaras, Fluid phase lipid areas and bilayer thicknesses of commonly used phosphatidylcholines as a function of temperature, Biochimica et Biophysica Acta (BBA)-Biomembranes 1808 (11) (2011) 2761-2771. 
[26] J. P. Jämbeck, A. P. Lyubartsev, Derivation and systematic validation of a refined all-atom force field for phosphatidylcholine lipids, The journal of physical chemistry B 116 (10) (2012) 3164-3179.

[27] J. Daillant, E. Bellet-Amalric, A. Braslau, T. Charitat, G. Fragneto, F. Graner, S. Mora, F. Rieutord, B. Stidder, Structure and fluctuations of a single floating lipid bilayer, Proceedings of the National Academy of Sciences of the United States of America 102 (33) (2005) 11639-11644.

[28] S. Shenoy, P. Shekhar, F. Heinrich, M.-C. Daou, A. Gericke, A. H. Ross, M. Lösche, Membrane association of the pten tumor suppressor: molecular details of the protein-membrane complex from spr binding studies and neutron reflection, PloS one 7 (4) (2012) e32591.

[29] S. S. Shenoy, H. Nanda, M. Lösche, Membrane association of the pten tumor suppressor: electrostatic interaction with phosphatidylserinecontaining bilayers and regulatory role of the c-terminal tail, Journal of structural biology 180 (3) (2012) 394-408, (**) MD simulations help interpret NR data on membrane-associated protein and provide distributions of specific protein and lipid subunits.

[30] G. T. Tietjen, Z. Gong, C.-H. Chen, E. Vargas, J. E. Crooks, K. D. Cao, C. T. Heffern, J. M. Henderson, M. Meron, B. Lin, et al., Molecular mechanism for differential recognition of membrane phosphatidylserine by the immune regulatory receptor tim4, Proceedings of the National Academy of Sciences 111 (15) (2014) E1463-E1472.

[31] G. T. Tietjen, J. L. Baylon, D. Kerr, Z. Gong, J. M. Henderson, C. T. Heffern, M. Meron, B. Lin, M. L. Schlossman, E. J. Adams, E. Tajkhorshid, K. Y. C. Lee, Coupling x-ray reflectivity and in silico binding to yield dynamics of membrane recognition by tim1, Biophysical journal 113 (7) (2017) 1505-1519, (**) Complementary use of XRR and MD simulations provides detailed information on the configuration of a membrane-bound protein. 
[32] I. Rodriguez-Loureiro, V. M. Latza, G. Fragneto, E. Schneck, Conformation of single and interacting lipopolysaccharide surfaces bearing o-side chains, Biophysical journal 114 (7) (2018) 1624-1635.

[33] E. Schneck, E. Papp-Szabo, B. E. Quinn, O. V. Konovalov, T. J. Beveridge, D. A. Pink, M. Tanaka, Calcium ions induce collapse of charged O-side chains of lipopolysaccharides from Pseudomonas aeruginosa., J. R. Soc. Interface 6 Suppl 5 (Suppl 5) (2009) S671-8, (**) Combination of XRR experiments and CGMC simulations elucidates the response of a bacterial outer surface model to divalent cations.

[34] J. Daillant, L. Bosio, J. Benattar, C. Blot, Interaction of cations with a fatty acid monolayer. a grazing incidence x-ray fluorescence and reflectivity study, Langmuir 7 (4) (1991) 611-614.

[35] V. Padmanabhan, J. Daillant, L. Belloni, S. Mora, M. Alba, O. Konovalov, Specific ion adsorption and short-range interactions at the air aqueous solution interface, Physical review letters 99 (8) (2007) 086105.

[36] E. Schneck, T. Schubert, O. V. Konovalov, B. E. Quinn, T. Gutsmann, K. Brandenburg, R. G. Oliveira, D. A. Pink, M. Tanaka, Quantitative determination of ion distributions in bacterial lipopolysaccharide membranes by grazing-incidence $\mathrm{x}$-ray fluorescence, Proceedings of the National Academy of Sciences 107 (20) (2010) 9147-9151, (*) Combination of TRXF experiments and CGMC simulations elucidates ion distributions at mimics of bacterial surfaces. .

[37] M. Bedzyk, D. Bilderback, G. Bommarito, M. Caffrey, J. Schildkraut, Xray standing waves: a molecular yardstick for biological membranes, Science 241 (4874) (1988) 1788-1791.

[38] E. Schneck, E. Scoppola, J. Drnec, C. Mocuta, R. Felici, D. Novikov, G. Fragneto, J. Daillant, Atom-scale depth localization of biologically important chemical elements in molecular layers, Proceedings of the National Academy of Sciences 113 (34) (2016) 9521-9526. 
[39] J. Als-Nielsen, D. Jacquemain, K. Kjaer, F. Leveiller, M. Lahav, L. Leiserowitz, Principles and applications of grazing incidence x-ray and neutron scattering from ordered molecular monolayers at the air-water interface, Physics Reports 246 (5) (1994) 251-313.

[40] R. G. Oliveira, E. Schneck, B. E. Quinn, O. V. Konovalov, K. Brandenburg, T. Gutsmann, T. Gill, C. B. Hanna, D. A. Pink, M. Tanaka, Crucial roles of charged saccharide moieties in survival of gram negative bacteria against protamine revealed by combination of grazing incidence $\mathrm{x}$-ray structural characterizations and Monte Carlo simulations, Phys. Rev. E Stat. Nonlinear, Soft Matter Phys. 81 (4) (2010) 1-12.

[41] C. Stefaniu, I. Vilotijevic, M. Santer, D. Varón Silva, G. Brezesinski, P. H. Seeberger, Subgel phase structure in monolayers of glycosylphosphatidylinositol glycolipids, Angewandte Chemie International Edition 51 (51) (2012) 12874-12878.

[42] W. Abuillan, A. Vorobiev, A. Hartel, N. G. Jones, M. Engstler, M. Tanaka, Quantitative determination of the lateral density and intermolecular correlation between proteins anchored on the membrane surfaces using grazing incidence small-angle $\mathrm{x}$-ray scattering and grazing incidence $\mathrm{x}$-ray fluorescence, The Journal of Chemical Physics 137 (20) (2012) 204907.

[43] H. Ahrens, K. Graf, C. Helm, Observation of a superstructure x-ray peak within lipopolymer monolayers on the water surface, Langmuir 17 (11) (2001) 3113-3115.

[44] T. Salditt, Thermal fluctuations and stability of solid-supported lipid membranes, Journal of Physics: Condensed Matter 17 (6) (2005) R287.

[45] E. Schneck, B. Demé, C. Gege, M. Tanaka, Membrane adhesion via homophilic saccharide-saccharide interactions investigated by neutron scattering, Biophysical journal 100 (9) (2011) 2151-2159. 
[46] W. Pfeiffer, S. König, J. Legrand, T. Bayerl, D. Richter, E. Sackmann, Neutron spin echo study of membrane undulations in lipid multibilayers, EPL (Europhysics Letters) 23 (6) (1993) 457.

[47] M. Doxastakis, V. G. Sakai, S. Ohtake, J. Maranas, J. De Pablo, A molecular view of melting in anhydrous phospholipidic membranes, Biophysical journal 92 (1) (2007) 147-161.

[48] M. C. Rheinstädter, J. Das, E. J. Flenner, B. Brüning, T. Seydel, I. Kosztin, Motional coherence in fluid phospholipid membranes, Physical review letters 101 (24) (2008) 248106.

[49] T. R. Weikl, D. Andelman, S. Komura, R. Lipowsky, Adhesion of membranes with competing specific and generic interactions, The European Physical Journal E 8 (1) (2002) 59-66.

[50] J. Hu, T. Weikl, R. Lipowsky, Vesicles with multiple membrane domains, Soft Matter 7 (13) (2011) 6092-6102.

[51] M. Mohammadi, S. Shahhosseini, M. Bayat, Direct numerical simulation of water droplet coalescence in the oil, International Journal of Heat and Fluid Flow 36 (2012) 58-71.

[52] D. Pink, L. Truelstrup Hansen, T. Gill, B. E. Quinn, M. Jericho, T. Beveridge, Divalent calcium ions inhibit the penetration of protamine through the polysaccharide brush of the outer membrane of gram-negative bacteria, Langmuir 19 (21) (2003) 8852-8858.

[53] S. R. Midtgaard, M. C. Pedersen, J. J. K. Kirkensgaard, K. K. Sørensen, K. Mortensen, K. J. Jensen, L. Arleth, Self-assembling peptides form nanodiscs that stabilize membrane proteins, Soft Matter 10 (5) (2014) 738-752, (*) Combination of SAXS/SANS and CG simulations elucidates self assembly and structure of lipid/peptide nanodiscs. 
[54] S. J. Marrink, A. H. De Vries, A. E. Mark, Coarse grained model for semiquantitative lipid simulations, The Journal of Physical Chemistry B 108 (2) (2004) 750-760.

[55] J. Sun, D. Bousquet, H. Forbert, D. Marx, Glycine in aqueous solution: solvation shells, interfacial water, and vibrational spectroscopy from ab initio molecular dynamics, The Journal of chemical physics 133 (11) (2010) $09 \mathrm{~B} 609$.

[56] G. S. Grest, M. Murat, Structure of grafted polymeric brushes in solvents of varying quality: a molecular dynamics study, Macromolecules 26 (12) (1993) 3108-3117.

[57] T. Kreer, M. Müser, K. Binder, J. Klein, Frictional drag mechanisms between polymer-bearing surfaces, Langmuir 17 (25) (2001) 7804-7813.

[58] D. Dimitrov, A. Milchev, K. Binder, Polymer brushes in solvents of variable quality: Molecular dynamics simulations using explicit solvent, The Journal of chemical physics 127 (8) (2007) 084905.

[59] F. Léonforte, M. Müller, Poly (n-isopropylacrylamide)-based mixed brushes: a computer simulation study, ACS applied materials \& interfaces 7 (23) (2015) 12450-12462.

[60] R.-X. Gu, H. I. Ingolfsson, A. H. de Vries, S. J. Marrink, D. P. Tieleman, Ganglioside-lipid and ganglioside-protein interactions revealed by coarsegrained and atomistic molecular dynamics simulations, The Journal of Physical Chemistry B 121 (15) (2016) 3262-3275.

[61] H. Shiba, H. Noguchi, Estimation of the bending rigidity and spontaneous curvature of fluid membranes in simulations, Physical Review E 84 (3) (2011) 031926.

[62] V. Knecht, S.-J. Marrink, Molecular dynamics simulations of lipid vesicle fusion in atomic detail, Biophysical journal 92 (12) (2007) 4254-4261. 
[63] J. Chanda, S. Bandyopadhyay, Molecular dynamics study of surfactant monolayers adsorbed at the oil/water and air/water interfaces, The Journal of Physical Chemistry B 110 (46) (2006) 23482-23488.

[64] D. Horinek, A. Herz, L. Vrbka, F. Sedlmeier, S. I. Mamatkulov, R. R. Netz, Specific ion adsorption at the air/water interface: The role of hydrophobic solvation, Chemical Physics Letters 479 (4) (2009) 173-183.

[65] M. Kanduč, A. Schlaich, A. H. De Vries, J. Jouhet, E. Maréchal, B. Demé, R. R. Netz, E. Schneck, Tight cohesion between glycolipid membranes results from balanced water-headgroup interactions, Nature Communications 8.

[66] C. M. Shepherd, K. A. Schaus, H. J. Vogel, A. H. Juffer, Molecular dynamics study of peptide-bilayer adsorption, Biophysical journal 80 (2) (2001) $579-596$.

[67] W. F. van Gunsteren, J. Dolenc, A. E. Mark, Molecular simulation as an aid to experimentalists, Current opinion in structural biology 18 (2) (2008) 149-153.

[68] Y. Kang, S. Barbirz, R. Lipowsky, M. Santer, Conformational diversity of o-antigen polysaccharides of the gram-negative bacterium shigella flexneri serotype y, The Journal of Physical Chemistry B 118 (9) (2014) 2523-2534.

[69] F. Sedlmeier, D. Horinek, R. R. Netz, Spatial correlations of density and structural fluctuations in liquid water: A comparative simulation study, Journal of the American Chemical Society 133 (5) (2011) 1391-1398.

[70] L. Boldon, F. Laliberte, L. Liu, Review of the fundamental theories behind small angle x-ray scattering, molecular dynamics simulations, and relevant integrated application, Nano reviews 6 (1) (2015) 25661.

[71] A. Soper, Partial structure factors from disordered materials diffraction data: An approach using empirical potential structure refinement, Physical Review B 72 (10) (2005) 104204. 
[72] A. C. Jensen, S. Imberti, S. F. Parker, E. Schneck, Y. Politi, P. Fratzl, L. Bertinetti, W. J. Habraken, Hydrogen bonding in amorphous calcium carbonate and molecular reorientation induced by dehydration, The Journal of Physical Chemistry C.

[73] M. Fukuto, B. Ocko, D. J. Bonthuis, R. Netz, H.-G. Steinrück, D. Pontoni, I. Kuzmenko, J. Haddad, M. Deutsch, Nanoscale structure of the oil-water interface, Physical review letters 117 (25) (2016) 256102, (**) Combination of XRR and MD simulations elucidates the density profile across oil-water interfaces. .

[74] S. Schöttl, J. Marcus, O. Diat, D. Touraud, W. Kunz, T. Zemb, D. Horinek, Emergence of surfactant-free micelles from ternary solutions, Chemical Science 5 (8) (2014) 2949-2954.

[75] K. Binder, A. Milchev, Polymer brushes on flat and curved surfaces: How computer simulations can help to test theories and to interpret experiments, Journal of Polymer Science Part B: Polymer Physics 50 (22) (2012) 15151555 .

[76] G. Peters, N. B. Larsen, T. Bjørnholm, S. Toxvaerd, K. Schaumburg, K. Kjaer, X-ray diffraction and molecular-dynamics studies: Structural analysis of phases in diglyceride monolayers, Physical Review E 57 (3) (1998) 3153, (**) Combination of GIXD experiments and MD simulations reveals the nature of phase transitions in diglyceride monolayers.

[77] T. Salditt, C. Li, A. Spaar, U. Mennicke, X-ray reflectivity of solidsupported, multilamellar membranes, The European Physical Journal E 7 (2) (2002) 105-116, (*) Comparison between MD simulation and XRR data on lipid multilayers leads to a set of lipid bilayer structural parameters.

[78] N. Kučerka, D. Marquardt, T. A. Harroun, M.-P. Nieh, S. R. Wassall, D. H. de Jong, L. V. Schäfer, S. J. Marrink, J. Katsaras, Cholesterol in bilayers with pufa chains: doping with dmpc or popc results in sterol reorientation 
and membrane-domain formation, Biochemistry 49 (35) (2010) 7485-7493,

(*) Combination of neutron diffraction and MD simulations elucidates the configuration of cholesterol in lipid bilayers.

[79] R. G. Oliveira, E. Schneck, B. E. Quinn, O. V. Konovalov, K. Brandenburg, U. Seydel, T. Gill, C. B. Hanna, D. A. Pink, M. Tanaka, Physical mechanisms of bacterial survival revealed by combined grazing-incidence x-ray scattering and monte carlo simulation, Comptes Rendus Chimie 12 (1) (2009) 209-217, (*) Combination of XRR-equivalent experiments and CGMC simulations elucidates the influence of divalent cations on the resistance of bacteria against antimicrobial peptides.

[80] D. Constantin, U. Mennicke, C. Li, T. Salditt, Solid-supported lipid multilayers: structure factor and fluctuations, The European Physical Journal E 12 (2) (2003) 283-290.

[81] P. S. Coppock, J. T. Kindt, Determination of phase transition temperatures for atomistic models of lipids from temperature-dependent stripe domain growth kinetics, The Journal of Physical Chemistry B 114 (35) (2010) 11468-11473.

[82] J. B. Klauda, N. Kučerka, B. R. Brooks, R. W. Pastor, J. F. Nagle, Simulation-based methods for interpreting x-ray data from lipid bilayers, Biophysical journal 90 (8) (2006) 2796-2807, (**) Combination of x-ray diffraction and MD simulations yields meaningful density profiles of lipid bilayers.

[83] J. C. Fogarty, M. Arjunwadkar, S. A. Pandit, J. Pan, Atomically detailed lipid bilayer models for the interpretation of small angle neutron and $\mathrm{x}$ ray scattering data, Biochimica et Biophysica Acta (BBA)-Biomembranes 1848 (2) (2015) 662-672.

[84] O. S. Ollila, G. Pabst, Atomistic resolution structure and dynamics of lipid bilayers in simulations and experiments, Biochimica et Biophysica Acta (BBA)-Biomembranes 1858 (10) (2016) 2512-2528. 
[85] J. S. Hub, T. Salditt, M. C. Rheinstädter, B. L. De Groot, Short-range order and collective dynamics of dmpc bilayers: a comparison between molecular dynamics simulations, x-ray, and neutron scattering experiments, Biophysical journal 93 (9) (2007) 3156-3168, (*) Combination of x-ray/neutron scattering and MD simulations elucidates lipid dynamics in bilayers.

[86] J. D. Nickels, X. Cheng, B. Mostofian, C. Stanley, B. Lindner, F. A. Heberle, S. Perticaroli, M. Feygenson, T. Egami, R. F. Standaert, J. C. Smith, D. A. A. Myles, M. Ohl, J. Katsaras, Mechanical properties of nanoscopic lipid domains, Journal of the American Chemical Society 137 (50) (2015) 15772-15780, (**) Combination of SANS, inelastic neutron scattering, and atomistic simulations provides insight into structure and mechanics of nanodomains in unilamellar vesicles. 